\title{
Supplementary material \\ Bootstrap-based procedures for inference in nonparametric receiver-operating characteristic curve regression analysis
}

\author{
María Xosé Rodríguez-Álvarez ${ }^{1,2,3}$, Javier Roca-Pardiñas ${ }^{1}$, \\ Carmen Cadarso-Suárez ${ }^{4}$ and Pablo G. Tahoces ${ }^{5}$ \\ ${ }^{1}$ Department of Statistics and Operations Research \\ and Biomedical Research Centre (CINBIO), University of Vigo, Vigo, Spain \\ ${ }^{2}$ BCAM - Basque Center for Applied Mathematics, Bilbao, Spain \\ ${ }^{3}$ IKERBASQUE, Basque Foundation for Science, Bilbao, Spain \\ ${ }^{4}$ Center for Research in Molecular Medicine and Chronic Diseases (CiMUS), \\ University of Santiago de Compostela, Santiago de Compostela, Spain \\ ${ }^{5}$ Centro Singular de Investigación en Tecnologías de la Información (CITIUS), \\ University of Santiago de Compostela, Santiago de Compostela, Spain
}

This document contains supplementary material to the paper "Bootstrap-based procedures for inference in nonparametric receiver-operating characteristic curve regression analysis". Additional simulation studies to complete those presented in the main manuscript are provided. More precisely, we present here the results when considering different distributions for simulating the diagnostic test result in healthy and diseased populations, and when covariates only affect the result of the diagnostic test in the diseased population.

\section{Web Appendix A Simulation study with different distribu- tions}

Data were simulated from two scenarios, namely,

- Scenario wI

$$
\begin{aligned}
& Y_{\bar{D}}=-2 X_{v 1}^{2}+0.5 \exp \left(X_{v 2}\right)+\varepsilon_{\bar{D}} \\
& Y_{D}=a X_{v 1}^{2}-2 X_{v 1}^{2}+0.5 \sin \left(\pi\left(X_{v 2}+1\right)\right)+0.5 \exp \left(X_{v 2}\right)+\varepsilon_{D}
\end{aligned}
$$


- Scenario wII

$$
\begin{aligned}
& Y_{\bar{D}}=-0.25 X_{v 1}^{3}+0.5 X_{v 1}^{2}+0.5 X_{v 1}^{2} X_{u 1}-0.5 X_{v 1}^{2}\left(1-X_{u 1}\right)+\varepsilon_{\bar{D}} \\
& Y_{D}=0.25 X_{v 1}^{3}+(a+1)\left(0.5 X_{v 1}^{2}+0.5 X_{v 1}^{2} X_{u 1}-0.5 X_{v 1}^{2}\left(1-X_{u 1}\right)\right)+\varepsilon_{D} .
\end{aligned}
$$

In both cases, $a$ is a real constant, $X_{v 1}$ and $X_{v 2}$ are simulated from a uniform distribution on $[-1,1]$, and $X_{u 1} \sim$ Bernoulli (0.5).

Bearing in mind the distributions of errors $\varepsilon_{\bar{D}}$ and $\varepsilon_{D}$ the following situations are considered:

(a) $\varepsilon_{\bar{D}}$ and $\varepsilon_{D}$ displaying Student's $t$ distributions, both with a mean of zero and 12 degrees of freedom

(b) $\varepsilon_{\bar{D}}$ displaying a Gaussian distribution with a mean of zero an a standard deviation of 0.5 , and $\varepsilon_{D}$ displaying a mixture of Gaussian distributions, $\varepsilon_{D} \sim 0.5 N\left(-0.5,0.5^{2}\right)+$ $0.5 N\left(0.5,0.5^{2}\right)$

With the above configurations, the corresponding conditional ROC curves under the assumption of Student's $t$ distributed errors are

- Scenario wIa

$$
\operatorname{ROC}_{\boldsymbol{x}}(p)=T_{12}\left(a x_{v 1}^{2}+0.5 \sin \left(\pi\left(x_{v 2}+1\right)\right)+T_{12}^{-1}(p)\right) .
$$

- Scenario wIIa

$$
R O C_{\boldsymbol{x}}(p)=T_{12}\left(0.5 x_{v 1}^{3}+0.5 a\left(x_{v 1}^{2}+x_{v 1}^{2} x_{u 1}-x_{v 1}^{2}\left(1-x_{u 1}\right)\right)+T_{12}^{-1}(p)\right) .
$$

where $T_{d f}$ denotes the cumulative distribution function for the standard Student's $t$ distribution with $d f$ degrees of freedom. For the situation of mixture of Gaussian distributions, the conditional ROC curve for each scenario considered is:

- Scenario wIa

$$
R O C_{\boldsymbol{x}}(p)=F\left(a x_{v 1}^{2}+0.5 \sin \left(\pi\left(x_{v 2}+1\right)\right)+0.5 \Phi^{-1}(p)\right) .
$$

- Scenario wIIa

$$
R O C_{\boldsymbol{x}}(p)=F\left(0.5 x_{v 1}^{3}+0.5 a\left(x_{v 1}^{2}+x_{v 1}^{2} x_{u 1}-x_{v 1}^{2}\left(1-x_{u 1}\right)\right)+0.5 \Phi^{-1}(p)\right) .
$$

where $\Phi$ denotes the cumulative distribution function of a standard normal random variable, and $F(c)=0.5 \Phi\left(\frac{c+0.5}{0.5}\right)+0.5 \Phi\left(\frac{c-0.5}{0.5}\right)$. 


\begin{tabular}{|c|c|c|c|c|c|c|c|c|c|}
\hline & \multicolumn{2}{|c|}{ Sample size } & \multirow[b]{2}{*}{ Test } & \multicolumn{5}{|c|}{ Level } & \multirow[b]{2}{*}{ KS $p$-value } \\
\hline & $n_{D}$ & $n_{\bar{D}}$ & & 0.01 & 0.05 & 0.10 & 0.15 & 0.20 & \\
\hline \multirow{6}{*}{ Scenario wIa } & \multirow{2}{*}{50} & \multirow{2}{*}{50} & 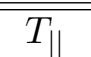 & "0.013 & 0.052 & 0.102 & 0.156 & 0.219 & 0.687 \\
\hline & & & $T_{2}$ & 0.013 & 0.058 & 0.113 & 0.168 & 0.220 & 0.407 \\
\hline & \multirow{2}{*}{200} & \multirow{2}{*}{200} & $T_{\|}$ & 0.015 & 0.051 & 0.112 & 0.159 & 0.205 & 0.007 \\
\hline & & & $T_{2}$ & 0.013 & 0.051 & 0.114 & 0.166 & 0.206 & 0.006 \\
\hline & \multirow{2}{*}{1000} & \multirow{2}{*}{1000} & $T_{\|}$ & 0.008 & 0.038 & 0.091 & 0.143 & 0.186 & 0.117 \\
\hline & & & $T_{2}$ & 0.009 & 0.049 & 0.087 & 0.138 & 0.187 & 0.087 \\
\hline \multirow{6}{*}{ Scenario wIb } & \multirow{2}{*}{50} & \multirow{2}{*}{50} & $S_{\|}$ & 0.005 & 0.032 & 0.096 & 0.132 & 0.182 & 0.064 \\
\hline & & & $S_{2}^{\prime \prime}$ & 0.004 & 0.031 & 0.085 & 0.138 & 0.178 & 0.063 \\
\hline & \multirow{2}{*}{200} & \multirow{2}{*}{200} & $S_{\|}$ & 0.008 & 0.049 & 0.106 & 0.150 & 0.189 & 0.192 \\
\hline & & & $S_{2}$ & 0.011 & 0.043 & 0.093 & 0.152 & 0.193 & 0.149 \\
\hline & \multirow{2}{*}{1000} & \multirow{2}{*}{1000} & $S_{\|}$ & 0.014 & 0.058 & 0.103 & 0.136 & 0.183 & 0.468 \\
\hline & & & $S_{2}$ & 0.017 & 0.054 & 0.100 & 0.141 & 0.180 & 0.263 \\
\hline \multirow{6}{*}{ Scenario wIIa } & \multirow{2}{*}{50} & \multirow{2}{*}{50} & $S_{\|}$ & 0.011 & 0.047 & 0.087 & 0.143 & 0.193 & 0.001 \\
\hline & & & $S_{2}$ & 0.010 & 0.046 & 0.096 & 0.143 & 0.182 & 0.004 \\
\hline & \multirow{2}{*}{200} & \multirow{2}{*}{200} & $S_{\|}$ & 0.009 & 0.046 & 0.098 & 0.155 & 0.200 & 0.811 \\
\hline & & & $S_{2}$ & 0.007 & 0.042 & 0.096 & 0.148 & 0.195 & 0.909 \\
\hline & \multirow{2}{*}{1000} & \multirow{2}{*}{1000} & $S_{\|}$ & 0.014 & 0.053 & 0.095 & 0.141 & 0.180 & 0.055 \\
\hline & & & $S_{2}$ & 0.011 & 0.047 & 0.097 & 0.130 & 0.184 & 0.063 \\
\hline \multirow{6}{*}{ Scenario wIIb } & \multirow{2}{*}{50} & \multirow{2}{*}{50} & $S_{\|}$ & 0.014 & 0.040 & 0.082 & 0.123 & 0.168 & 0.001 \\
\hline & & & $S_{2}$ & 0.014 & 0.038 & 0.083 & 0.124 & 0.166 & 0.002 \\
\hline & \multirow{2}{*}{200} & \multirow{2}{*}{200} & $S_{\|}$ & 0.012 & 0.050 & 0.082 & 0.124 & 0.185 & 0.253 \\
\hline & & & $S_{2}$ & 0.011 & 0.043 & 0.087 & 0.133 & 0.183 & 0.272 \\
\hline & \multirow{2}{*}{1000} & 100 & $S_{\|}$ & 0.009 & 0.040 & 0.082 & 0.134 & 0.194 & 0.406 \\
\hline & & 1000 & $S_{2}$ & 0.009 & 0.037 & 0.082 & 0.133 & 0.187 & 0.405 \\
\hline
\end{tabular}

Web Table 1: For Scenarios wIa and wIIa (Student's $t$ distributions) and wIb and wIIb (mixture of Gaussian distributions): estimated type I error registered by the proposed tests under the null hypothesis, for different significance levels and sample sizes. The last column presents the $p$-values of the Kolmogorov-Smirnov test for uniformity of the observed $p$-values.

Table 1 shows the type I errors registered by the proposed tests for Scenarios wI and wII, for different significance levels and sample sizes. Figure 1 depicts quantile-quantile plots of the expected $p$-values (under the uniform distribution) and the observed $p$-values. As can be seen, the tests perform well in general, with type I errors proving to be relatively close to nominal errors (Table 3), and $p$-value distributions close to the uniform one (Figure 1). Table 2 shows the power of the tests at different significance levels for a specific value of $a$. As expected, the probability of rejection rises as the sample size increases. 

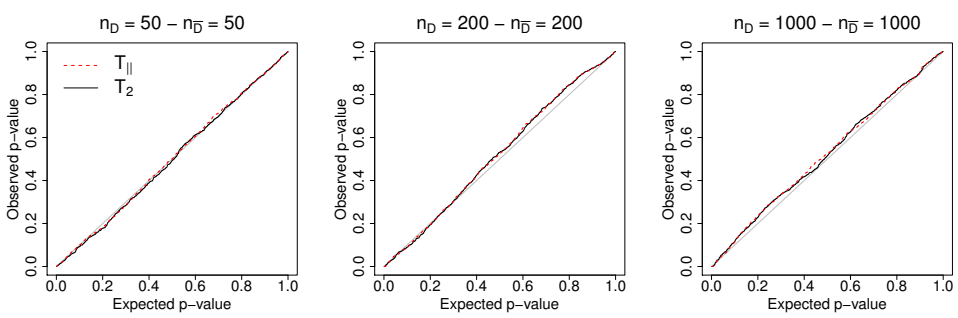

(a) Scenario wIa
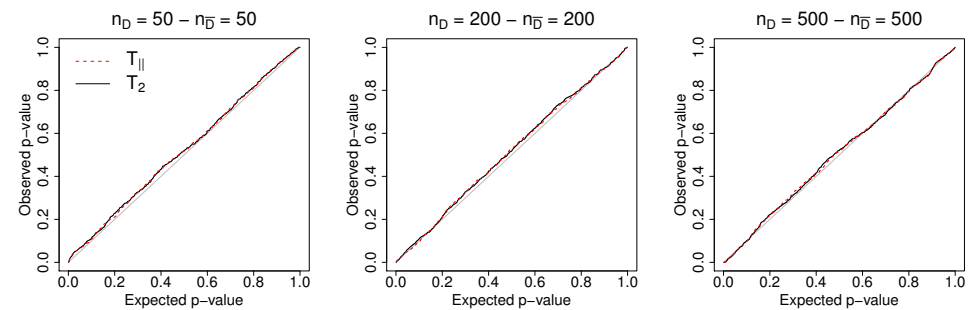

(b) Scenario wIb
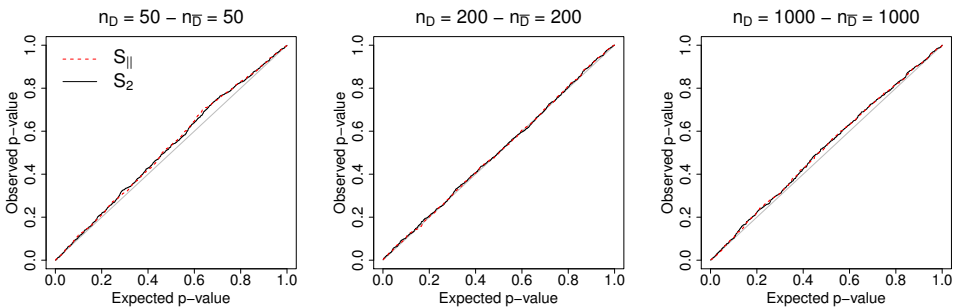

(c) Scenario wIIa
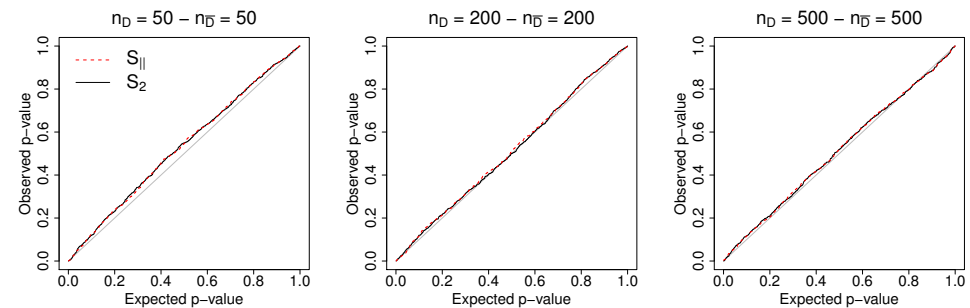

(d) Scenario wIIb

Web Figure 1: For Scenarios wIa and wIIa (Student's $t$ distributions) and wIb and wIIb (mixture of Gaussian distributions): Quantile-quantile plot for the the observed p-values vs the expected p-values when the null hypothesis is correct. 


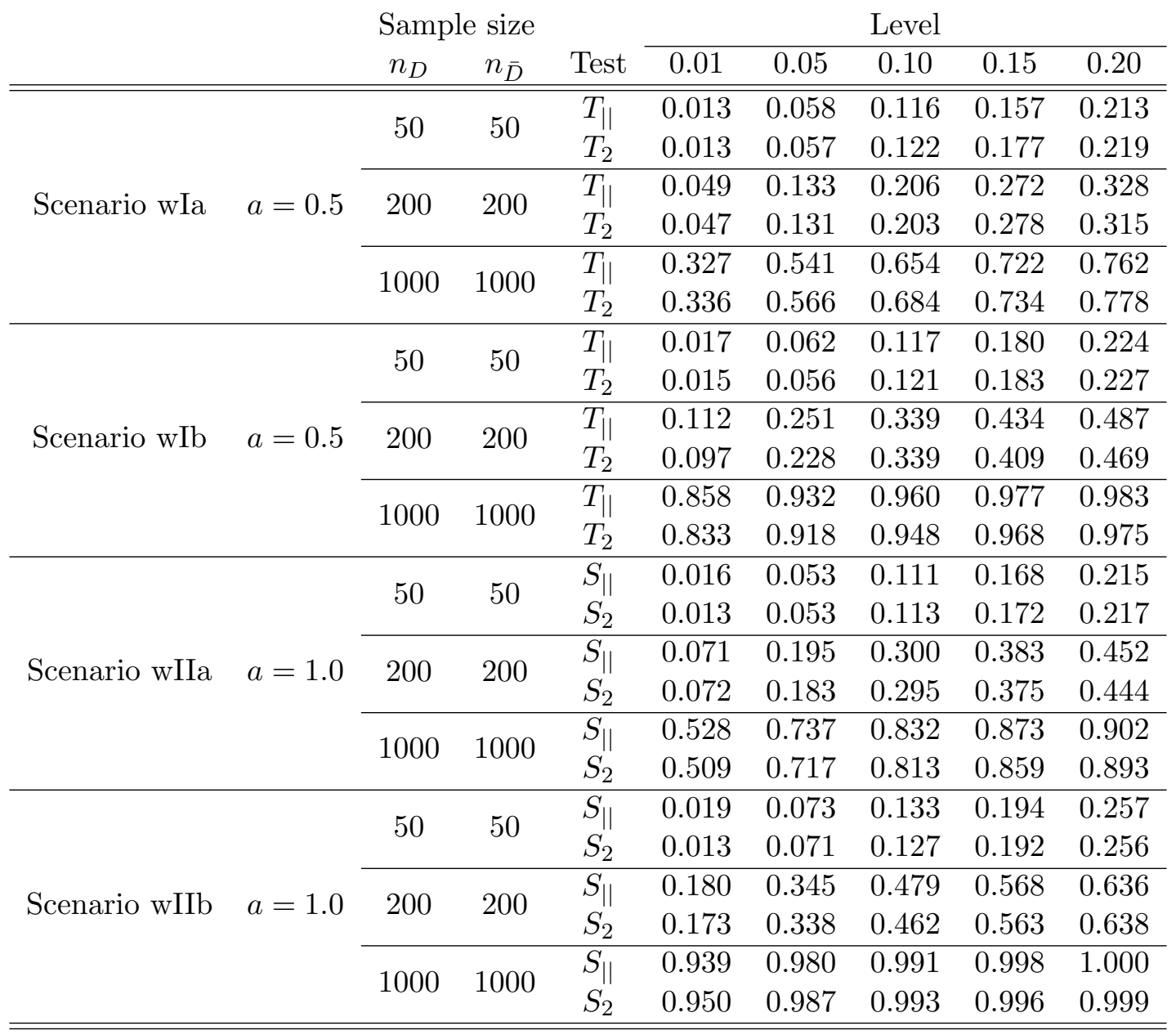

Web Table 2: For Scenarios wIa and wIIa (Student's $t$ distributions) and wIb and wIIb (mixture of Gaussian distributions): estimated rejection probabilities registered by the proposed tests under the alternative hypothesis, for different significance levels and sample sizes.

\section{Web Appendix B Simulation study with covariates affecting only the diseased population}

Data were simulated from two scenarios, namely,

- Scenario wIII

$$
\begin{aligned}
& Y_{\bar{D}}=0.5 \exp \left(X_{v 2}\right)+0.5 \varepsilon_{\bar{D}} \\
& Y_{D}=0.5 \sin \left(\pi\left(X_{v 2}+1\right)\right)+0.5 \exp \left(X_{v 2}\right)+a X_{v 1}^{2}+0.5 \varepsilon_{D} .
\end{aligned}
$$


- Scenario wIV

$$
\begin{aligned}
& Y_{\bar{D}}=-0.25 X_{v 1}^{3}+0.5 \varepsilon_{\bar{D}} \\
& Y_{D}=0.25 X_{v 1}^{3}+a X_{v 1}^{2} X_{u 1}+0.5 \varepsilon_{D}
\end{aligned}
$$

In both cases, $a$ is a real constant, $X_{v 1}$ and $X_{v 2}$ are simulated from a uniform distribution on $[-1,1], X_{u 1} \sim \operatorname{Bernoulli}(0.5)$ and $\varepsilon_{\bar{D}}$ and $\varepsilon_{D} \sim N(0,1)$. Note that in Scenario wIII the continuous covariate $X_{v 1}$ only affects the result of the diagnostic test in the diseased population. In much the same way, in Scenario wIV is the categorical covariate $X_{u 1}$ the one affecting only the result of the diagnostic test in the diseased population. Here, $a=0$ corresponds to the hypothesis of no interaction between $X_{v 1}$ and $X_{u 1}$, and as the value of $a$ rises, so does the degree of interaction.

With the above configurations, the corresponding conditional ROC curves are

- Scenario wIII

$$
\operatorname{ROC}_{\boldsymbol{x}}(p)=\Phi\left(2 a x_{v 1}^{2}+\sin \left(\pi\left(x_{v 1}+1\right)\right)+\Phi^{-1}(p)\right)
$$

- Scenario wIV

$$
\operatorname{ROC}_{\boldsymbol{x}}(p)=\Phi\left(x_{v 1}^{3}+a\left(x_{v 1}^{2}+x_{v 1}^{2} x_{u 1}-x_{v 1}^{2}\left(1-x_{u 1}\right)\right)+\Phi^{-1}(p)\right) .
$$

Table 3 shows the type I errors registered by the proposed tests for Scenarios wIII and wIV, for different significance levels and sample sizes. Figure 2 depicts quantile-quantile plots of the expected $p$-values (under the uniform distribution) and the observed $p$-values. As can be seen, the tests perform well in general, with type I errors proving to be relatively close to nominal errors (Table 3), and $p$-value distributions close to the uniform one (Figure 2 ). Table 4 shows the power of the tests at different significance levels for a specific value of $a$. As expected, the probability of rejection rises as the sample size increases. 


\begin{tabular}{|c|c|c|c|c|c|c|c|c|c|}
\hline & \multicolumn{2}{|c|}{ Sample size } & \multirow[b]{2}{*}{ Test } & \multicolumn{5}{|c|}{ Level } & \multirow[b]{2}{*}{ KS $p$-value } \\
\hline & $n_{D}$ & $n_{\bar{D}}$ & & 0.01 & 0.05 & 0.10 & 0.15 & 0.20 & \\
\hline \multirow{6}{*}{ Scenario wIII } & \multirow{2}{*}{50} & \multirow{2}{*}{50} & $\overline{T_{\|}}$ & 0.012 & 0.054 & 0.089 & 0.141 & 0.198 & 0.211 \\
\hline & & & $T_{2}$ & 0.011 & 0.049 & 0.094 & 0.145 & 0.192 & 0.185 \\
\hline & \multirow{2}{*}{200} & \multirow{2}{*}{200} & $T_{\|}$ & 0.007 & 0.055 & 0.101 & 0.142 & 0.191 & 0.289 \\
\hline & & & $T_{2}$ & 0.012 & 0.054 & 0.094 & 0.138 & 0.197 & 0.326 \\
\hline & \multirow{2}{*}{1000} & \multirow{2}{*}{1000} & $T_{\|}$ & 0.011 & 0.051 & 0.102 & 0.153 & 0.203 & 0.956 \\
\hline & & & $T_{2}$ & 0.014 & 0.050 & 0.107 & 0.153 & 0.206 & 0.741 \\
\hline \multirow{6}{*}{ Scenario wIV } & \multirow{2}{*}{50} & \multirow{2}{*}{50} & $S_{\|}$ & 0.006 & 0.052 & 0.114 & 0.156 & 0.197 & 0.882 \\
\hline & & & $S_{2}$ & 0.012 & 0.047 & 0.102 & 0.154 & 0.202 & 0.901 \\
\hline & \multirow{2}{*}{200} & \multirow{2}{*}{200} & $S_{\|}$ & 0.010 & 0.048 & 0.100 & 0.148 & 0.203 & 0.899 \\
\hline & & & $S_{2}$ & 0.015 & 0.053 & 0.109 & 0.148 & 0.200 & 0.550 \\
\hline & \multirow{2}{*}{1000} & \multirow{2}{*}{1000} & $S_{\|}$ & 0.009 & 0.052 & 0.098 & 0.149 & 0.194 & 0.363 \\
\hline & & & $S_{2}$ & 0.011 & 0.048 & 0.096 & 0.151 & 0.202 & 0.404 \\
\hline
\end{tabular}

Web Table 3: For Scenarios wIII and wIV (covariates affecting only the diseased population): estimated type I error registered by the proposed tests under the null hypothesis, for different significance levels and sample sizes. The last column presents the $p$-values of the Kolmogorov-Smirnov test for uniformity of the observed $p$-values.

\begin{tabular}{|c|c|c|c|c|c|c|c|c|c|}
\hline & & \multicolumn{2}{|c|}{ Sample size } & \multirow[b]{2}{*}{ Test } & \multicolumn{5}{|c|}{ Level } \\
\hline & & $n_{D}$ & $n_{\bar{D}}$ & & 0.01 & 0.05 & 0.10 & 0.15 & 0.20 \\
\hline \multirow{6}{*}{ Scenario wIII } & \multirow{6}{*}{$a=0.5$} & \multirow{2}{*}{50} & \multirow{2}{*}{50} & $\overline{T_{\|}}$ & "0.066 & 0.179 & $\overline{0.274}$ & 0.349 & 0.416 \\
\hline & & & & $T_{2}$ & 0.051 & 0.171 & 0.278 & 0.353 & 0.401 \\
\hline & & \multirow{2}{*}{200} & \multirow{2}{*}{200} & $T_{\|}$ & 0.636 & 0.821 & 0.879 & 0.914 & 0.933 \\
\hline & & & & $T_{2}$ & 0.611 & 0.782 & 0.869 & 0.906 & 0.921 \\
\hline & & \multirow{2}{*}{1000} & \multirow{2}{*}{1000} & $T_{\|}$ & 1.000 & 1.000 & 1.000 & 1.000 & 1.000 \\
\hline & & & & $T_{2}$ & 1.000 & 1.000 & 1.000 & 1.000 & 1.000 \\
\hline \multirow{6}{*}{ Scenario wIV } & \multirow{6}{*}{$a=1.0$} & \multirow{2}{*}{50} & \multirow{2}{*}{50} & $S_{\|}$ & 0.061 & 0.143 & 0.241 & 0.314 & 0.384 \\
\hline & & & & $S_{2}$ & 0.058 & 0.139 & 0.218 & 0.303 & 0.374 \\
\hline & & \multirow{2}{*}{200} & \multirow{2}{*}{200} & $S_{\|}$ & 0.563 & 0.783 & 0.864 & 0.914 & 0.944 \\
\hline & & & & $S_{2}$ & 0.527 & 0.757 & 0.852 & 0.905 & 0.940 \\
\hline & & \multirow{2}{*}{1000} & \multirow{2}{*}{1000} & $S_{\|}$ & 1.000 & 1.000 & 1.000 & 1.000 & 1.000 \\
\hline & & & & $S_{2}$ & 1.000 & 1.000 & 1.000 & 1.000 & 1.000 \\
\hline
\end{tabular}

Web Table 4: For Scenarios wIII and wIV (covariates affecting only the diseased population): estimated rejection probabilities registered by the proposed tests under the alternative hypothesis, for different significance levels and sample sizes. 

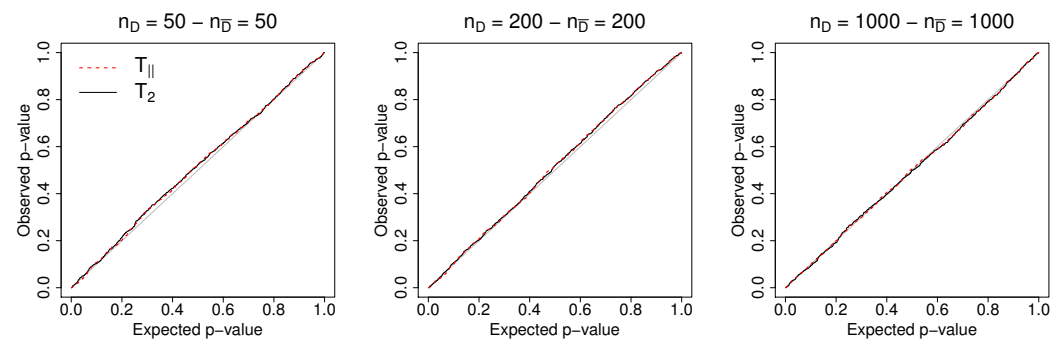

(a) Scenario wIII
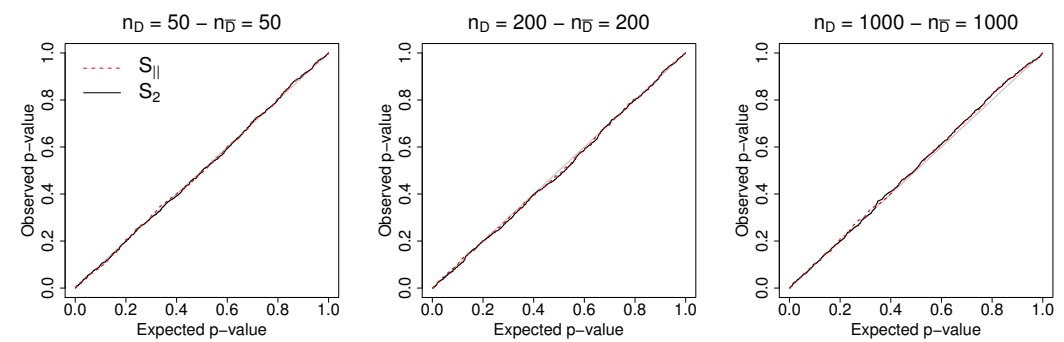

(b) Scenario wIV

Web Figure 2: For Scenarios wIII and wIV (covariates affecting only the diseased population): Quantile-quantile plot for the the observed p-values vs the expected p-values when the null hypothesis is correct. 